\title{
Criminologie
}

\section{Relations professionnelles en établissement pénitentiaire}

\section{Valérie Moulin et Anne-Sophie Sévin}

Volume 43, numéro 1, printemps-été 2010

Les jeunes et la rue

URI : https://id.erudit.org/iderudit/044058ar

DOI : https://doi.org/10.7202/044058ar

Aller au sommaire du numéro

Éditeur(s)

Les Presses de l'Université de Montréal

ISSN

0316-0041 (imprimé)

1492-1367 (numérique)

Découvrir la revue

Citer cet article

Moulin, V. \& Sévin, A.-S. (2010). Relations professionnelles en établissement pénitentiaire. Criminologie, 43(1), 227-248. https://doi.org/10.7202/044058ar

\section{Résumé de l'article}

Partant du constat d'une tendance au retrait des personnels de la zone de détention, en établissement pénitentiaire, au profit de l'investissement de postes qui ne les mettent pas en présence directe des détenus, cette recherche vise la compréhension de ce phénomène. Plus précisément, la recherche à partir de laquelle a été rédigé cet article a consisté à analyser ce qui génère de la conflictualité psychique chez les surveillants dans l'exercice de leur profession. À partir d'une modélisation de la conflictualité psychique dans l'exercice professionnel sur les coursives en établissements pénitentiaires, cet article dégage quatre positionnements professionnels des surveillants en maisons d'arrêt et leur fonction sur le plan psychique. 


\title{
Relations professionnelles en établissement pénitentiaire
}

\author{
Valérie Moulin \\ Maître de conférences en psychologie pathologique et criminologique \\ Laboratoire de Cliniques Psychologiques, Psychopathologie et Institut de Criminologie et Sciences \\ humaines, GIS CrimSo \\ Université de Rennes II \\ valerie.moulin@uhb.fr \\ Anne-Sophie Sévin \\ Psychologue clinicienne \\ Institut de Criminologie et Sciences humaines, GIS CrimSo \\ Université de Rennes II \\ anne.sophie.sevin@free.fr
}

RÉSUMÉ - Partant du constat d'une tendance au retrait des personnels de la zone de détention, en établissement pénitentiaire, au profit de l'investissement de postes qui ne les mettent pas en présence directe des détenus, cette recherche vise la compréhension de ce phénomène. Plus précisément, la recherche à partir de laquelle a été rédigé cet article a consisté à analyser ce qui génère de la conflictualité psychique chez les surveillants dans l'exercice de leur profession. À partir d'une modélisation de la conflictualité psychique dans l'exercice professionnel sur les coursives en établissements pénitentiaires, cet article dégage quatre positionnements professionnels des surveillants en maisons d'arrêt et leur fonction sur le plan psychique.

MOTS CLÉS • Établissement pénitentiaire, surveillant, conflictualité psychique, positionnement professionnel.

\section{Introduction}

Périodiquement, dans des moments de crise ou de grandes découvertes, ou lors de la sortie d'un ouvrage (Vasseur, 2000), la société française s'indigne sur l'état de ses prisons, «pour l'oublier aussitôt» :

Il faut lire l'étonnement et la surprise actuelle face à la prison contemporaine comme résultante d'un défaut de mémoire de nos sociétés. Elle 
se révèle être un des lieux les plus soumis à l'amnésie sociale. En ce sens, on peut dire qu'elle est un trou de mémoire; de même qu'un ancien détenu doit masquer ses années de prison sur un curriculum vitae, de même nous faisons en sorte de gommer le plus possible le problème des prisons dans la vie sociale. (Arthieres et Lascoumes, 2004)

Si la prison est géographiquement localisable au sein de la ville, socialement reconnue, culturellement et idéologiquement acceptée, elle n'en demeure pas moins l'objet de représentations, sentiments, fantasmes et projections les plus contradictoires, recouvrant partiellement ces «lieux autres», «hétérotopiques» décrits par Foucault.

Nonobstant l'évolution des pratiques professionnelles pénitentiaires et de l'image du milieu carcéral durant ces quinze dernières années, la prison est sans cesse montrée du doigt et dénoncée par la presse lorsqu'un événement fâcheux arrive en détention (Rambourg, 2004). Cette image sociale dévalorisée ${ }^{1}$ touche aussi ceux qui y travaillent. Le pouvoir d'enfermer et de punir légalisé et légitimé par le modèle social, mais «toujours suspecté d'illégitimité et d'arbitraire» (Veil et Lhuillier, 2000), peut placer celui qui le représente dans une position inconfortable. Dans une confusion entre pouvoir de «garde» et pouvoir de punir, notamment à travers le symbole fort que constituent les clefs, la figure du surveillant peut être l'objet de représentations et de fantasmes négatifs au sein de la société et chez ceux qui «expérimentent» l'établissement pénitentiaire. Malgré l'attribution, en 1958, d'un statut spécial assimilant les personnels pénitentiaires aux forces de l'ordre et de sécurité, le malaise identitaire des surveillants qui peinent à donner un sens à leur profession, se répète à travers les évolutions législatives et les axes politiques.

Dans cet article, nous nous intéressons aux pratiques professionnelles des agents de l'administration pénitentiaire qui travaillent sur les postes «coursives» en maison d'arrêt. Dans les établissements pénitentiaires français, les coursives constituent un lieu de passage et de circulation dans la détention, mais également un lieu de vie, donnant accès aux cellules et aux douches pour les détenus. Dans cet espace circulent et se rencontrent les détenus, les agents, les gradés et différents personnels pénitentiaires, ainsi que les intervenants extérieurs. Pour le surveillant, les coursives représentent l'espace du collectif et le lieu de rencontre avec l'autre, principalement le détenu. Les maisons d'arrêt faisant périodique-

1. Qui est notamment le résultat de son histoire et de la tâche primaire de l'institution. 
ment l'objet d'une surpopulation carcérale, elles constituent des espaces restreints qui se caractérisent par le flux des détenus ou leur absence à certaines heures de la journée. L’Administration pénitentiaire définit comme suit le poste des surveillants sur les coursives:

L'agent [...] est en contact direct et constant avec les détenus. Il maintient l'ordre et la discipline et s'assure du respect du règlement intérieur de l'établissement. Il participe activement à la prévention des incidents et des évasions mais reste aussi l'interlocuteur privilégié du détenu dans la relation quotidienne. Il est le premier interlocuteur du détenu et participe activement à son observation dans le cadre notamment du projet d'application des peines. (Ministère de la justice (2008), Pratiques de Références opérationnelles, PRO: 9)

Les coursives en maison d'arrêt n'apparaissent plus comme l'espace privilégié de déploiement de l'activité professionnelle du surveillant, ce qui nous a amenées à investiguer les raisons pour lesquelles les agents quittent cet espace de travail pour d'autres postes tels que l'infirmerie, le parloir, le quartier mineur, la porte. Ce travail ${ }^{2}$ a consisté à analyser ce qui génère de la conflictualité psychique chez les surveillants lorsqu'ils travaillent sur les coursives.

Considérant le milieu carcéral comme un système complexe (Morin et Le Moigne, 1999; Le Moigne, 1999), l'objectif de ce travail est de dégager, à partir d'une analyse contextuelle et contextualisée, les situations qui conflictualisent et/ou mettent en difficulté les professionnels dans l'espace coursives en maison d'arrêt; puis les processus psychiques que cela met en jeu et qui peuvent s'exprimer par des positionnements professionnels différentiels («manière d'être en situation professionnelle»); ainsi que leurs fonctions dans l'espace en question et dans l'institution. Cet article vise uniquement à présenter les quatre positionnements professionnels dégagés. La conflictualité est à envisager selon une double facette comme pouvant être constructive, porteuse d'ouverture et de créativité, mais aussi vulnérabilisante lorsqu'elle conduit à des difficultés professionnelles, voire personnelles.

Après avoir présenté succinctement les axes d'interrogation et de modélisation de l'objet de recherche, nous présentons quatre positionnements professionnels dégagés dans l'espace coursives en maison

2. Il s'agit d'une recherche plus large que la partie restituée dans cet article. Cet article rapporte uniquement les résultats concernant les positionnements professionnels dans l'espace coursives. 
d'arrêt. Les résultats présentés sont le fruit d'un travail de recherche mené dans trois établissements pénitentiaires français, auprès du personnel de l'Administration pénitentiaire.

\section{Axes de conflictualisation des positions professionnelles}

Une analyse de la littérature et des travaux empiriques existants associée à une phase exploratoire en établissement nous ont conduites à dégager six principaux axes de conflictualisation potentielle des positionnements professionnels ${ }^{3}$ des surveillants.

\section{Axe relationnel, soi-autrui}

Ce qui apparaît prégnant dans un établissement pénitentiaire est la pluralité des relations, qui constituent la «matière première» de l'exercice professionnel du surveillant. Il s'agit de surcroît d'une relation sous contrainte légale et réglementaire, dissymétrique (Veil et Lhuillier, 2000) et dans laquelle les positions de surveillant et de surveillé, de gardant et de gardé, demeurent non interchangeables. Ces éléments participent au maintien du cadre et de l'équilibre institutionnel.

La spécificité des relations en milieu carcéral et leur importance quantitative nous ont conduites à interroger tout particulièrement l'axe relationnel et les relations soi-autrui, qu'il s'agisse bien sûr des relations entre les surveillants et les détenus, mais aussi avec les collègues de l'institution et les intervenants extérieurs qui traversent ou investissent des espaces de l'établissement pénitentiaire. Ce premier axe d'analyse caractérise la manière d'être d'un sujet en situation et relation; en particulier en situation professionnelle. Mais également sa capacité à se positionner comme sujet singulier et à entrer en relation avec autrui de manière différenciée. Il s'agira de repérer ce qui, dans le rapport à l'autre, peut conflictualiser une position professionnelle et/ou personnelle, mais également être porteur d'altérité et de reconnaissance des différences, des places et des rôles de chacun.

Dans les situations difficiles, le groupe de pairs des surveillants par sa cohésion, sa reconnaissance, sa valorisation joue un rôle fondamen-

3. Ce modèle peut être transposé dans d'autres types d'institutions, d'établissements. 
tal de facteur d'étayage ${ }^{4}$, nécessaire à la stabilité identitaire du surveillant, mais également à sa stabilité psychique dans les situations vulnérantes (agression, conflits, violences) et dans le quotidien du maintien d'une relation sous contrainte. Lorsque l'institution ou le groupe de pairs ne sont plus suffisamment soutenants pour le surveillant, le sujet peut mobiliser des stratégies défensives nécessaires au maintien de sa stabilité psychique. Stratégies qui peuvent consister en des formes d'attaques de l'institution (Kaës, 2000; Enriquez, 2000) lorsque cette dernière ne soutient plus ses sujets, et ne les protège pas du danger. L'origine commune des sujets dans l'institution se fonde dans l'adhésion narcissique à «l'objet-institution»; si le sujet se sent abandonné par l'institution, le contrat narcissique qui le lie à cet objet et fonde le mythe et le discours institutionnel est fragilisé, voire rompu. Cela peut prendre chez le surveillant la forme d'abandon professionnel ou de discours dévalorisant et de représentations dépréciées à l'égard de l'institution perçue comme maltraitante, qui instrumentalise les professionnels, etc.

\section{Axe légalité-légitimité}

La question de la légalité renvoie au rapport à la loi et à la règle, à ce qui est explicitement édicté par le Code pénal et le règlement intérieur. La légalité peut se définir comme ce qui est conforme au droit. Dans la profession de surveillant, il s'agit du cadre légal et réglementaire qui circonscrit l'exercice du métier ${ }^{5}$; la transgression de la loi ou de la règle est susceptible d'impliquer des sanctions pénales et disciplinaires. Quant à la légitimité, elle exprime le rapport du sujet aux valeurs et aux normes individuelles et groupales, à la constitution d'une morale individuelle et d'une éthique au regard de ces éléments. Les valeurs individuelles vont constituer la dimension éthique de la personne. L'éthique édicte un certain nombre de principes de la conduite humaine à respecter, elle aura des incidences en termes d'actions, de réflexions et de positions personnelles et professionnelles, individuelles ou groupales. Elle impose à chaque personne, prise dans sa singularité, des interdits qui, lorsqu'ils sont transgressés, peuvent entraîner un sentiment

4. Le terme étayage est utilisé en référence à la théorie freudienne et signifie «s'appuyer sur». Ce terme est utilisé en psychologie dans l'analyse de la dynamique groupale.

5. Constitutif de certains éléments du cadre carcéral. 
de culpabilité; selon que la personne s'autorise et se permette de faire telle ou telle action, on parlera en termes de permission, d'autorisation, de légitimation, ou encore de légitimité.

Cette conflictualité sur l'axe légalité-légitimité peut par exemple apparaittre dans l'histoire de la profession ou dans certains paradoxes institutionnels. Les deux missions de garde et de réinsertion qui se trouvent au cœur du métier peuvent être sources de conflictualité pour l'agent (Mbanzoulou, 2000; Silberman, 1995). Sur le plan des paradoxes institutionnels, il est possible d'évoquer la tension entre l'application stricte de la loi et la nécessité de l'aménager (Chauvenet et al., 1994) afin de maintenir le calme et la sécurité en détention. Cette injonction paradoxale place le personnel de surveillance dans une posture de transgression entre légalité et légitimité, potentiellement source de conflictualité.

À côté des zones de conflictualisation potentielle, sur l'axe légalitélégitimité, le contexte carcéral peut induire des mises en conflit éthique dans le rapport du sujet à lui-même, lorsque valeurs personnelles et professionnelles s'affrontent dans des pratiques telles que «la fouille à corps $^{6} »$ par exemple; dans le fait de demeurer présent dans des relations intimes lors des parloirs, etc., autant d'éléments qui renvoient à la transgression d'interdits mettant en avant l'opposition entre sphères privée et publique; voire dans ce qui constitue le fondement même de la tâche primaire de l'institution, la garde; mais encore lorsque le surveillant se fait prendre au «piège de la compassion» (Goffman, 1968), etc.

\section{Axe axiologique, valeurs personnelles et valeurs du groupe}

Il s'agit ici d'appréhender les valeurs dominantes du groupe de pairs, ou groupe d'appartenance professionnelle; valeurs et éthique groupales qui seront véhiculées dans les discours, les manières d'être en situations et relations professionnelles ainsi que dans les usages dans la pratique professionnelle en établissement; la profession des surveillants se caractérise par la force et la cohésion du groupe des pairs, qui a une fonction contenante et étayante face aux différentes difficultés rencontrées. Les appartenances idéologiques, syndicales, culturelles, l'histoire institutionnelle (Mercier, 2004), la constitution d'un code de valeurs et

6. Expression utilisée dans l'administration pénitentiaire pour caractériser la pratique professionnelle qui consiste à fouiller les détenus. 
de langage peuvent avoir une incidence forte sur la manière d'être et peuvent être générateurs d'une «sous-culture des surveillants» (Carlier, 1997), de positionnements professionnels tranchés (Kauffman, 1988), voire de pressions professionnelles (Kauffman, op. cit.) ; éléments constitutifs d'un phénomène nommé "prisonniérisation» des surveillants (Vacheret et Lemire, 2007).

Les valeurs du groupe et leurs incidences sur la manière d'être et d'exercer ont ensuite été mises en perspective avec celle de l'agent interviewé, afin de repérer si cela peut être générateur de conflits.

\section{Axe autorité-pouvoir}

À l'intérieur ou à l'extérieur de la prison, l'autorité n'est plus reconnue et acceptée d'emblée. Il ne suffit plus de «porter l'uniforme» pour être reconnu comme «porteur d'autorité ${ }^{\natural}$. L'autorité est différente du pouvoir car elle s'exerce à travers la reconnaissance symbolique des places de chacun et au «nom d'une loi» supérieure à toutes les autres, dont les professionnels pénitentiaires sont dépositaires par mandat émanant du corps social et de l'autorité de l'État. Dans un espace légalement et institutionnellement réglementé et contraint, tel que la prison, lorsque les relations professionnelles avec les détenus ne peuvent plus s'exercer sous la forme d'un rapport d'autorité, elles peuvent glisser vers un rapport de pouvoir et de lutte de pouvoir; les relations professionnelles n'ayant plus la possibilité d'être régulées par des rapports symboliques, relatifs aux positions différentielles de chacun au sein de cet espace. $\mathrm{Si}$ l'autorité n'est plus soutenue par la fonction remplie ${ }^{8}$, le rapport à autrui ne peut s'exercer sous la forme d'un «droit d'imposition légitime» (Bourdieu, 1970) et se transforme en rapport de violence, vécu comme tel tant du côté des professionnels que des détenus, lorsqu'il n'y a plus de rempart symbolique pour médiatiser la rencontre.

Le pouvoir impliquerait la disparition de l'autre au profit de soi, dans une dynamique de maintien d'un équilibre psychique individuel (pouvant s'exprimer sous la forme du contrôle, de l'emprise, de la maîtrise, etc., dans la relation). L'autorité renvoie au droit d'imposition légal et légitime que peut exercer un sujet par rapport à un autre, au regard de la fonction remplie et occupée.

7. Que l'on fasse référence à la police, à la gendarmerie.

8. En tant que juge, policier, enseignant, parent. 


\section{Axe temporel}

Cet axe s'intéresse à la perception et au vécu temporel dans l'exercice professionnel, il permet de caractériser la temporalité du surveillant, tant qu'il s'agit du rapport singulier qu'un agent entretient avec la dimension temporelle, impliquant la distinction entre temps «objectif» et «subjectif». Le premier correspond à un temps normé, fixé et quantifié, le temps de l'horloge; il s'agit d'un «temps socialisé» (Lameyre, 2005), déterminé de l'extérieur, qui propose un cadre et participe à la formation des habitus sociaux. Dans le champ institutionnel, il prendra la forme des horaires de travail, des emplois du temps, de l'organisation et de la structuration du temps; temps plein et temps vide, temps «forts» et temps «morts». Le second correspond au temps «perçu, vécu»; il fait référence à la perception des durées et au vécu de l'écoulement du temps, relativement au rapport de l'homme au temps. Larticulation des temps «objectif et subjectif» est nécessaire à une «subjectivation existentielle» et professionnelle (Lameyre, 2005 ; Levy, 2007). Sans possibilité d'aménagements subjectifs du temps socialisé et construit par l'institution, le temps ne se structure plus et n'est plus structurant, il est perçu comme subi, sous une forme contrainte et n'offre plus de perspective «poiétique ${ }^{9} »$. Le temps et les temps institutionnels étant fortement investis et déterminants sur le plan institutionnel, l'organisation, l'autonomie, la contrainte, la liberté, le pouvoir (Foucault, 1975) s'y articulent directement.

\section{Axe spatial: interne-externe}

Cet axe d'analyse caractérise les rapports entre monde interne et monde externe, tant dans ce qui a trait à l'articulation entre espace professionnel et espace privé (Siret, 2004), en référence par exemple aux différentes formes possibles d'empiètement du monde professionnel sur le monde privé (et inversement) et les conflits que cela peut produire chez les surveillants; entre espace psychique interne et espace social. Par exemple, il s'agit ici d'observer si la vie professionnelle s'immisce dans la vie personnelle, voire psychique, et sous quelles modalités (exemple: penser répétitivement à certaines scènes professionnelles, etc.). Mais

9. Lameyre (2005) utilise ce terme en référence au grec ancien, dans lequel «poiétikos», signifie «capable de créer». 
également en raison de l'organisation spatiale des locaux d'exercice professionnel, la manière dont cela peut susciter du malaise, par exemple à travers l'absence d'espace de retrait et une proximité physique obligée entre détenus et surveillants, etc.

L'articulation de ces différents axes d'analyse a permis de modéliser comme suit l'objet de recherche.

F I G URE 1

Modélisation de l'objet de recherche

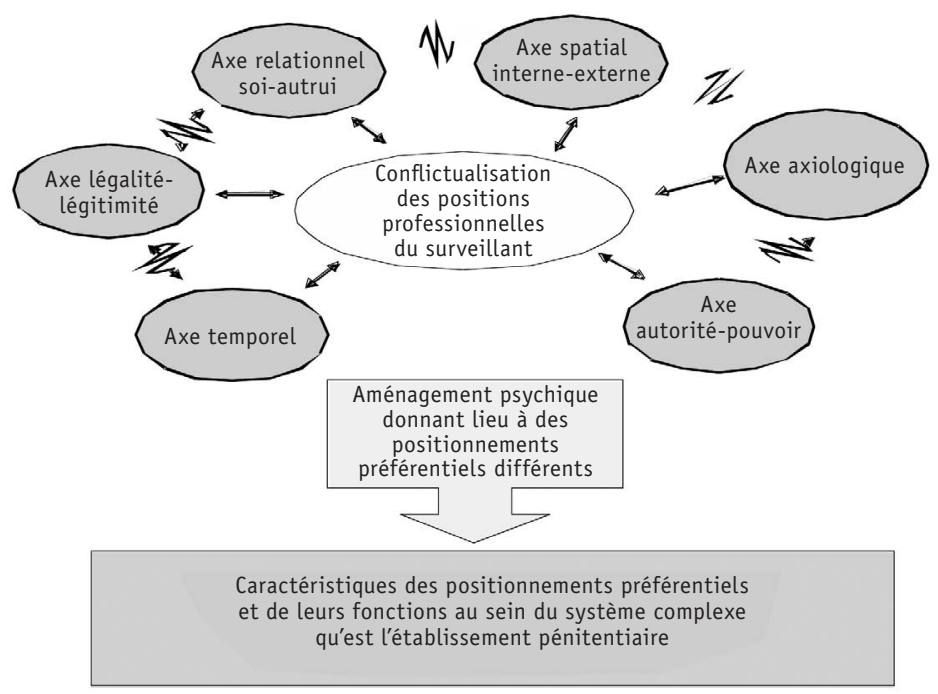

\section{Méthodologie de la recherche}

L'échantillon d'étude est composé de trois établissements pénitentiaires: une maison d'arrêt et deux centres pénitentiaires. La recherche s'est déroulée sur les trois sites d'étude, à partir d'un entretien semi-directif de recherche. Les résultats de cette recherche se basent sur 72 entretiens menés auprès du personnel pénitentiaire (d'une durée d'environ une heure et trente) et des observations participantes dans l'établissement. L'entretien semi-directif de recherche opérationnalise les axes d'étude à partir de dimensions et d'indicateurs qui rendent compte des positions professionnelles et des points de conflictualisation de celles-ci. 
Le lieu de travail, les coursives, a été analysé à partir des perceptions et vécus des surveillants. Nous avons investigué, puis analysé les dimensions suivantes: les représentations et discours associés aux coursives (sens donné aux différents espaces d'activité, représentation et vécu du temps de travail, temporalité, représentation des dimensions active ou passive de l'activité professionnelle, possibilité d'espace de retrait, etc.); les représentations et rapports aux détenus (représentation du détenu par le surveillant, gestion de la distance relationnelle, place dans la gestion du groupe, type de relation au détenu, etc.); les représentations et rapports aux collègues, la fonction du groupe; le rapport à la hiérarchie, à l'institution; les représentations et rapports aux non pénitentiaires $^{10}$; les représentations et rapports entre «mondes interne et externe $e^{11} »$.

Les éléments d'analyse présentés correspondent à la vision du personnel pénitentiaire. Il s'agit d'une approche phénoménologique qui vise à décrire, à partir des perceptions et des vécus, la façon dont l'agent appréhende son environnement, se positionne et réagit face à celui-ci.

\section{Positionnements professionnels dans l'espace coursives en maison d'arrêt}

À partir d'une analyse contextualisée, considérant les agents de l'administration pénitentiaire dans le milieu dans lequel ils évoluent comme des êtres en situation et en relation avec leur environnement, porteurs d'une histoire et d'une identité professionnelle et agissant à ce titre de manière particulière sur leur environnement, notre modèle permet de présenter des positionnements professionnels différentiels, une compréhension de ceux-ci et une analyse de leurs fonctions sur le plan psychique individuel et groupal. Les positionnements professionnels peuvent être considérés comme des aménagements possibles d'une position psychique, ayant pour fonction générale de rétablir l'équilibre dynamique de ce qui, potentiellement, peut être conflictualisé par l'exercice en milieu carcéral. Lorsque c'est le cas, les «manières d'être et d'exercer» peuvent être considérées comme des stratégies adaptatives qui permettent de minimiser, d'atténuer, voire d'éviter les contraintes dans l'activité professionnelle.

10. Les professionnels: médecins, avocats, psychiatres, instituteurs, etc. qui ne sont pas des agents pénitentiaires mais qui travaillent pour une autre institution.

11. En référence à la dimension spatiale. 
À l'instar de travaux existants relevant davantage d'une analyse sociologique (Montadon et Crettaz, 1981 ; Kauffman, 1988; Rostaing, 1997; Vacheret, 2002), notre étude met en évidence des positionnements axés sur la garde et la sécurité dans deux cas sur quatre et un positionnement davantage orienté vers les dimensions d'écoute, d'observation et une relation individualisée avec les détenus, que l'on peut globalement rapprocher d'un positionnement plus social, nommé «missionnaire» par Rostaing (1997). Un dernier positionnement renvoie à un désinvestissement du métier et de son exercice, dans une posture de retrait et de protection personnelle, qui se rapproche du type «fonctionnaire» de Kauffman (1988).

Positionnement professionnel caractérisé par un vécu de «trop-plein » et de suspicion

Ce qui ressort, dans ce type de positionnement, est la dimension de «trop-plein» qui se caractérise par l'importance des sollicitations qui émanent des détenus, pour lesquels le surveillant est le seul interlocuteur. Sollicité en permanence par les détenus, par le bruit ${ }^{12}$ (Claerhout, 2004), importuné par les odeurs et en raison de la pluralité des activités à accomplir dans des laps de temps très courts, le surveillant travaille selon un rythme tendu et gère des flux de détenus qui rendent difficile l'individualisation des rapports avec ces derniers. «Tout ce que tu as le temps de faire sur les coursives, c'est d'ouvrir et fermer les portes, emmener les gars d'un endroit à un autre et t'assurer que rien ne va dégénérer», "Tu n'as pas le temps de discuter ${ }^{13}$ ».

Cet espace est vécu sur le mode de la tension et du stress en raison de la pluralité des éléments à gérer et des risques sécuritaires, ce qui conduit les surveillants à être particulièrement vigilants. Cet espace apparaît comme un espace subi, qui assigne le surveillant à une posture de passivité face à ce trop-plein d'activités. Sans véritable maîtrise et dans une sensation de perte de contrôle face à la diversité des sollicitations et des tâches à réaliser, le surveillant met en place des stratégies pour prendre le contrôle de la situation dans des positionnements de pouvoir qui visent à préserver un équilibre psychique individuel.

12. Pour exprimer leurs demandes, les détenus appellent fréquemment en criant, en frappant contre les portes, etc.

13. Propos d'un surveillant; dans les citations suivantes, nous notons S pour le préciser. 
Labsence d'espace de retrait physique, associé à la dimension du panoptique, et le sentiment de perte de contrôle de cet espace alimentent des vécus d'envahissement.

Ce vécu, d'envahissement et de «trop plein», met à mal les capacités d'élaboration mentale des surveillants, c'est-à-dire les capacités à penser et à élaborer leurs émotions face non plus seulement à des événements que l'on qualifierait de traumatiques, mais au cumul et à la répétition de ce type de situations. C'est la dimension répétitive et l'incapacité à pouvoir élaborer ce vécu qui affectent les surveillants. Évoquant l'aspect quantitatif et cumulatif des événements, Diwo (1997) aborde la notion de «seuil»; quelles que soient les capacités de mentalisation des sujets, au-delà d'un certain seuil de cumul d'événements: «il y aurait faillite transitoire de l'appareil mental et "aiguillage", "bascule" vers d'autres voies, celles du recours à l'agir et (ou) de l'expression somatique» (Diwo, 1997:107). Les capacités d'élaboration mentale rendent compte du processus de transformation des excitations et des affects en contenus mentaux symbolisés, c'est-à-dire en expressions cognitives, représentations et pensées. Ces excitations sur le plan psychique, lorsqu'elles sont renouvelées et intenses, créent un trop-plein, une accumulation qui attaquent les processus de pensée: «les excitations doivent trouver des voies régulières d'écoulement, d'élaboration ou de décharge pour ne pas s'accumuler et devenir excessives, leurs accumulations s'avérant la cause majeure des somatisations» (Marty, 1996).

Proche de la «logique statutaire» décrite par Rostaing (1997), le rapport aux détenus est davantage centré sur les fonctions de surveillance, de garde et de contrôle, mettant en avant la nécessaire obéissance et soumission des détenus. Les surveillants se positionnent dans une relation de gestion de masse sans individualisation des rapports et de leur perception: «moi je dis le détenu, car dans le fond ils sont tous pareils» $(S)$. Face à une relation au détenu perçue sur un mode envahissant, d'abus et d'instrumentalisation, les stratégies mises en place pour mettre à distance l'autre et se protéger psychiquement correspondent à des attitudes qui consistent à faire attendre, à se retirer et ne pas répondre; à imposer, à interdire. Introduisant une logique de pouvoir au détriment d'une logique d'autorité et d'une régulation intersubjective des relations détenus-surveillants.

À côté d'un mouvement projectif du malaise des surveillants sur le groupe des détenus, qui s'en font les dépositaires, ce mécanisme est massivement observé à l'encontre de l'institution pénitentiaire, voire de 
la hiérarchie. Dans une forme de paranoïsation des liens institutionnels, l'institution revêt les traits d'un objet persécuteur qui ne protège plus ses personnels et avantage les détenus (dans une relation en miroir, où tout gain du côté des détenus est perçu comme une perte du coté du personnel), voire peut être vécue comme volontairement maltraitante à l'égard des surveillants. Mouvement qui peut être repris et porté par la voie syndicale: «La direction? On la voit quand il y a un problème, quand tu n'as pas fait ton boulot, mais quand il faut nous soutenir il n'y a plus personne» $(\mathrm{S})$.

Le groupe demeure étayant, rassurant et contenant face au malaise et aux angoisses; il existe des possibilités d'exutoire, voire de mise à distance grâce au groupe des pairs. L'articulation entre éthique personnelle et déontologie professionnelle/individuelle et valeurs groupales n'est pas génératrice de conflits pour l'individu. Les valeurs du groupe prennent le dessus sur les valeurs individuelles et conditionnent le positionnement professionnel, construit à partir de ces valeurs dominantes ${ }^{14}$. Lorsque l'éthique personnelle et la déontologie professionnelle ne sont plus en accord avec les valeurs du groupe, les agents sont amenés à demander des postes extérieurs car leur positionnement ne paraît plus soutenable eu égard à la pression du groupe de pairs et à la tendance à l'homogénéisation des modes de pensée et des positionnements professionnels. Les valeurs sécuritaires du groupe prennent le dessus sur les valeurs personnelles et donnent du sens à l'activité professionnelle dans cette perspective.

\section{Positionnement professionnel caractérisé par un vécu «d'envahissement» par la relation}

Les représentations et les discours associés à l'espace de travail sont similaires à ceux présentés précédemment, mettant en avant les dimensions de stress et de tension, le vécu de trop-plein et le ressenti de perte de contrôle des événements face au flux des détenus et à la pluralité des sollicitations. Ce qui apparaît prégnant ici concerne les dimensions de contamination et de contagion par le bruit, l'odeur, la faute, la proximité et l'intimité des détenus, alimentant le sentiment de vivre «au milieu des rebus de la société, ceux que personne ne veut» (S). Comme si le

14. Phénomène de prisonniérisation des détenus, cette fois du côté du personnel pénitentiaire (Vacheret et Lemire, 2007). 
surveillant n'était plus «étanche» et se laissait envahir par ces différentes dimensions, mettant en évidence la problématique de la séparation et des limites. Les barrières sur le plan psychique n'apparaissent plus protectrices.

Comme précédemment, la relation avec les détenus se présente sous la forme d'une gestion des flux dans une posture non individualisée et de distance. Le positionnement professionnel, marqué par une rigidification des règles et du cadre, ne peut souffrir d'aménagements qui seraient vécus comme dangereux psychiquement, impliquant un rapprochement, à l'instar d'une relation individualisée qui mobilise des rapports de proximité. L'accent est mis sur une position statutaire (Rostaing, 1997), rigide dans la relation aux détenus, qui est vécue sur le mode de l'envahissement physique et psychique.

L'institution et la hiérarchie ne sont plus perçues comme contenantes, elles ne parviennent pas à réguler le malaise et le vécu de menace sur le plan psychique. Elles sont alors vécues comme maltraitantes et indifférentes au sort de leurs agents et à leur malaise, alimentant une position victimale et le sentiment de constituer une catégorie sacrifiée: "Ils savent nous rappeler à l'ordre et nous utiliser quand ils ont besoin, c'est tout, on est des pions» $(S)$. La direction peut être mise en cause pour son manque de rigueur, dans un appel au cadre structurant et contenant, à travers notamment la demande d'application stricte du règlement comme moyen de restaurer de la distance et de la différence dans la relation aux détenus.

Si le groupe de pairs garde une importance, il ne parvient plus à maintenir une dimension protectrice: des récriminations apparaissent, notamment autour de l'hétérogénéité des pratiques, qui est vécue comme problématique, car susceptible de remettre en question la cohésion et la force du groupe des surveillants dans le rapport (de force) aux détenus, et de mettre en péril leur légitimité face à ces derniers. Il ressort des entretiens le besoin de «faire corps et masse» contre le groupe des détenus qui est vécu comme «bloc homogène» face aux surveillants ${ }^{15}$.

Les barrières protectrices sur le plan psychique n'apparaissent plus suffisantes, elles suscitent des risques sur le plan individuel et groupal. Dans une impossible régulation de la proximité physique et psychique vécue sur le mode de l'envahissement, les surveillants mettent en place

15. Le port de l'uniforme est évoqué en ces termes. 
des stratégies défensives sur le plan psychique à travers la rigidification des règles et du cadre, comme moyen de réintroduire des limites, du tiers et de la distance, dans une posture sécuritaire marquée. Les dimensions d'écoute, de proximité et d'individualisation, davantage associées à la réinsertion, sont difficiles à tenir car elles alimentent le rapprochement et le vécu d'un risque de contagion, de contamination, etc. La dévalorisation des détenus, leur perception en tant qu'individus dangereux sont là aussi autant de moyens de tenter de réguler sur un mode imaginaire une distance à l'autre qui ne peut plus l'être dans les rapports quotidiens de la détention (les mécanismes de projection, clivage, paranoïsation des liens institutionnels sont également présents): «Il y a eux et il y a nous, et ce qui est usant, c'est de toujours leur rappeler de rester à leur place»; "faut pas confondre, ici c'est chacun de son côté» $(S)$; «on n'est pas pareil eux et nous, un détenu çà reste un détenu, faut garder ses distances» $(S)$. Ce positionnement professionnel a pour fonction principale de restaurer des limites et de la distance entre soi et l'autre, car sans cesse mises à mal. L'élaboration «d'une théorie de la nature humaine» (Goffman, 1968) justifie des positionnements radicaux et rigides ainsi qu'un mode de valorisation professionnelle à travers des postures très sécuritaires et la question de la dangerosité. Le surveillant peut alors se focaliser sur la mission de garde et évacuer la mission de réinsertion qui n'a plus, ici, aucun sens pour lui (Mbanzoulou, 2000). Cette perception du détenu et de la fonction professionnelle permet de spécifier la nature de l'exercice et le type de relation à entretenir avec les détenus, donnant ainsi sens à l'activité professionnelle tout en se protégeant et en construisant une représentation positive de soi, notamment valeureuse de celui qui «garde» des individus dangereux.

Dans ce groupe, troubles somatiques et addictifs sont prévalents, ainsi que les arrêts-maladie comme modalité pour "faire coupure» (Siret, 2004), l'instauration de lieux sas et la nécessité d'introduire une barrière étanche entre vie privée et vie professionnelle «afin de ne pas ramener la taule chez soi» $(S)$.

\section{Positionnement professionnel marqué par une logique de désinvestissement et de retrait}

Ce qui apparaît, ici, le plus prégnant est le non-sens de l'activité professionnelle; les discours mettent en avant les dimensions de vide, d'ennui, d'insatisfaction et d'inutilité dans l'exercice professionnel du 
surveillant. Non-sens de la profession, non-sens des missions qui légitiment un discours et un positionnement professionnel de désinvestissement: «Ici tu ne sers à rien, tu ouvres et tu fermes les portes, et puis la majorité des gars qu'on voit passer reviennent» $(S)$.

Face aux différentes problématiques présentées dans l'espace coursives en maison d'arrêt, les surveillants optent pour un retrait protecteur dans un processus psychologique de l'ordre de la dénégation, de l'isolation, voire du déni, «ne plus vouloir voir ou entendre» ce qui se déroule en détention et dans l'établissement pénitentiaire. Il s'agit ici de ne pas se laisser envahir par les problèmes de la détention, par son rythme de travail, par les sollicitations, par le bruit, etc., optant pour un positionnement professionnel permissif à l'égard des détenus de l'ordre du «laisser-faire», de la fuite face aux tâches à réaliser qui sont reportées à plus tard ou laissées à un collègue, ou encore sous la forme d'une absence de réponse dans une logique d'abandon qui vise à se préserver.

Cette mise «hors champ de conscience» des problématiques de la détention est contrebalancée par un investissement privilégié de la vie personnelle: les horaires et activités professionnelles sont, autant que faire se peut, calquées sur la vie extérieure, valorisant cette dernière au détriment de la profession; la volonté de changer de poste est justifiée par la réalisation d'activités extérieures et l'adéquation du temps de travail à ces dernières. La demande de poste fixe hors détention est souvent mise en avant comme stratégie adaptative face à ce vécu.

La relation aux détenus se base sur un «moindre» contact possible, dans une posture de retrait (Montandon et Crettaz, 1981); il s'agit de minimiser les réponses, voire de refuser de répondre aux sollicitations, dans certains cas, sans raison valable. Il ressort des entretiens le souhait de se retirer du contact avec les détenus aussi souvent que possible, par la recherche d'espaces dépourvus de toute relation (miradors, horaires de nuit...).

Principalement dans une dynamique de gestion des flux ${ }^{16}$, sans posture individualisée, permettant une non-implication dans les relations humaines, le rapport aux détenus se présente principalement sous la forme d'un rapport de pouvoir dans une protection individuelle, où la spécificité et la singularité de l'autre ne sont pas reconnues et acceptées: «C'est toi qui décides et qui as raison, c'est toi qui es du bon côté,

16. La gestion des flux et la non-implication dans les relations permettent une neutralisation du rapport humain, fonctionnant comme un mécanisme d'isolation. 
pas le détenu, faut pas confondre, alors les règles ça se discute pas, c'est comme ça, tu t'économises» $(\mathrm{S})$.

Contrairement aux autres positionnements professionnels dans lesquels l'institution ${ }^{17}$ est présente sous différentes facettes dans les discours, elle disparaît ici dans une forme de désinvestissement professionnel et d'indifférence au fonctionnement institutionnel. Ce désinvestissement est opéré tout en veillant à ne pas attirer l'attention sur soi; demeurer discret pour «ne rien avoir à se reprocher» (S) face à la hiérarchie ou à la direction. Il s'agit d'un retrait vigilant quant à ses manifestations, toujours dans une logique de «ne pas être embêté»: «Je fais mes heures et je veux les passer tranquillement, ce qui importe c'est après, quand je sors et rentre chez moi» $(S)$.

Si le groupe peut potentiellement être étayant dans la mesure où il reçoit les plaintes, la plupart du temps apparaît une indifférence aux préoccupations et revendications du groupe de pairs. Éventuellement investi, pour les moments de convivialité, dans une logique individuelle, le groupe n'est plus porteur des idéaux et valeurs du surveillant, qui s'en distancie; apparaît une absence d'adhésion et d'intérêt pour ses revendications et ses mouvements.

Si les problématiques précédemment évoquées (trop-plein entraînant des processus de déliaison, mauvaise gestion de la distance relationnelle, phénomène d'envahissement, d'empiètement, contamination, contagion, etc.) sont présentes ici, elles suscitent des aménagements psychiques de l'ordre de la dénégation, du déni et un désinvestissement professionnel.

\section{Positionnement professionnel marqué par un investissement relationnel}

Ce positionnement est très minoritaire dans l'espace coursives.

Toujours perçu comme un espace où le surveillant doit être constamment vigilant, quant au maintien du cadre et de la sécurité, le temps est vécu sur le mode d'un «temps plein», rythmé et scandé par les tâches à réaliser. La diversité des tâches est valorisée sans qu'il n'y ait de vécu d'envahissement et la sensation de perdre le contrôle. Le surveillant vit son activité sous la forme d'un positionnement actif et maîtrisé. Les missions de garde et de réinsertion ne sont plus contradictoires.

17. Direction de l'établissement, Direction interrégionale ou Direction de l'administration pénitentiaire. 
Concernant la relation avec les détenus, les agents présentent un discours nuancé, valorisant l'instauration de relations individualisées, fondées sur une attitude empathique avec la volonté de comprendre la problématique des détenus, lorsque la gestion du temps et des flux le permet. Ils mettent en avant à la fois les problèmes de surpopulation carcérale, tout en privilégiant une relation d'écoute lorsque celle-ci est possible: «Il n'y a pas de hasard, à partir du moment où vous montrez de l'intérêt et du respect au détenu en face, vous lui faites comprendre que vous l'avez compris, alors ça détend, nous on peut bosser et se faire respecter et eux, ils sont plus à l'aise» $(S)$. Contrairement aux premiers groupes, les sollicitations viennent donner du sens à la profession et ne sont pas vécues sur le mode de l'envahissement. La plupart des sujets constitutifs de ce groupe demandent à sortir des coursives pour investir des postes fixes, qui leur permettraient de travailler davantage la relation: "On est un peu mariés aux détenus, c'est une vie commune dans la journée, si on trouve un équilibre ça va, et comme dans un couple ça pète aussi parfois quand il n'y a plus de confiance ou qu'un des deux a dérapé» $(S)$.

Les surveillants expriment l'image dévalorisée de leur profession sur le plan social et un malaise lié à cette image négative, qu'ils souhaitent voir ou faire évoluer. Leurs récriminations à l'égard de la direction sont principalement dans ce registre, face à une direction qui, selon eux, ne les protège pas de cette image et ne fait rien pour quelle évolue.

À l'opposé des premiers positionnements, la hiérarchie et le cadre sont perçus comme contenant et étayant; les agents ont le sentiment d'être écoutés, soutenus en cas de difficultés dans l'exercice professionnel; mais aussi le sentiment d'être reconnus dans leur fonction. L'institution, quant à elle, est perçue comme indifférente au statut des surveillants, elle ne les inciterait pas à évoluer et leur offrirait peu de perspectives, malgré le malaise de nombreux agents.

L'hétérogénéité des pratiques, malgré le maintient d'un cadre cohérent, ne laisse apparaître aucune faille dans la mission de sécurité, qui est valorisée. Les surveillants expriment une prise de distance et un détachement par rapport aux valeurs du groupe de pairs; ils accordent une importance aux valeurs personnelles et un intérêt pour la dimension d'insertion et les relations individualisées.

La prise de distance avec le groupe permet de porter un regard critique sur le poids de ce dernier et l'homogénéisation des pratiques et des discours sur la profession: «Avec les années ça change pas, c'est 
les collègues qui participent aussi de cette mauvaise image de notre métier à dire qu'on fait un métier de merde» $(S)$.

Malgré ce positionnement, les dimensions de solidarité, de soutien, de cohésion avec les pairs sont toujours très présentes dans leurs discours et caractérisent les représentations des liens dans le groupe.

Si le vécu professionnel de ce groupe est relativement positif, il n'en demeure pas moins la nécessité pour les agents de faire coupure entre le milieu carcéral et le milieu social, «de ne pas emporter avec soi la détention et les problèmes d'ici» $(S)$, par l'instauration de «sas ${ }^{18}$ » (trajet en voiture, vestiaire...), l'exercice d'une activité physique de «décompression», ou toute autre forme d'exutoire physique.

\section{Conclusion}

Dans l'espace coursives en maison d'arrêt, le vécu de l'environnement professionnel du surveillant peut apparaître vulnérabilisant (Moulin, 2008), il n'apporte plus les repères nécessaires à la gestion des relations et entraine la mise en œuvre de différents processus psychiques par lesquels les sujets tentent de réguler le malaise, les souffrances.

L'espace coursives est un espace collectif, spécifié par l'absence d'espace à soi ou lieu de repli, il expose le surveillant au regard des autres (espace panoptique), c'est un espace de distorsion du vécu relationnel (vécu d'instrumentalisation et d'objectalisation ${ }^{19}$ par le surveillant), un espace du trop-plein (d'autres, de mouvements, de bruits...), de relations en face-à-face sans média ou médiateur qui alimente les relations en miroir avec les détenus dans une position de similarité, parfois insupportable. Ces différents éléments fragilisent psychologiquement les limites soi/autrui et alimentent le développement de processus de contagion, de contamination (par la faute par exemple); de processus de projection sur les détenus et sur la hiérarchie du malaise; mais aussi la création de boucs émissaires (Girard, 1982) qui deviennent les porteurs des maux, en permettant de se décharger. Les points précédents suscitent aussi la recherche de protection du côté du groupe à travers l'homogénéisation des pensées et des pratiques et la projection d'une représentation idéalisée, qui entretient l'illusion groupale... Tous

18. Un sas se définit comme une pièce dotée de deux portes étanches qui permettent le passage entre deux milieux dont les pressions sont différentes. Il s'agit ici, d'un espace intermédiaire qui agit comme «sas» entre milieu professionnel et personnel.

19. Au sens psychopathologique du terme. 
ces processus sont autant de moyens de trouver un sens ou un objet au malaise, mais également de réintroduire de la distance et de la différence avec les détenus, de se protéger psychiquement des effets de l'organisation architecturale des lieux, des phénomènes de groupe (gestion des flux et des masses) et des particularités de l'aménagement de la relation sous contrainte dans cet espace du collectif de la détention.

Si la question du malaise des surveillants dans l'exercice de leurs fonctions et celle du sens de leur profession n'est pas des problèmes nouveaux (Chauvenet et al., 2005 20), notre étude, construite dans une perspective comparative ${ }^{21}$, a permis de travailler ces points en fonction de différents espaces de travail en établissement pénitentiaire. Dès lors que l'activité professionnelle du surveillant peut être médiatisée et signifiée par un sens autre que celui de la garde et le maintien de la sécurité, elle permet au surveillant d'investir la profession de manière valorisante et sécurisante; le professionnel n'est plus pris dans le faceà-face de la relation, il décale son identité grâce à l'existence d'un objectif autre (éducation, soins...), par la symbolique du lieu (exemple, un lieu de soins), par les intervenants qui y exercent, par les échanges et grâce à la pluridisciplinarité. Ces différents éléments permettent d'introduire du tiers dans la relation surveillant-détenu, une meilleure gestion de la distance relationnelle, favorable à l'investissement professionnel; ils rendent possible l'articulation des missions de garde et de réinsertion dans des postures individualisées.

\section{Références}

Arthieres, P. H., \& Lascoumes, P. (2004). Gouverner, enfermer: la prison, un modèle indépassable? Paris: Presses de science politique.

Aymard, N., \& Lhuillier, D. (1993). Sécurité et identité professionnelle des personnels de surveillance. Rapport de recherche. Paris: GERAL.

Bourdieu, P. (1970). La reproduction: Éléments pour une théorie du système d'enseignement. Paris : Éditions de Minuit.

Carlier, C. (1996). Histoire du personnel des prisons françaises du XVIII siècle à nos jours. Paris: Éditions de l'atelier.

Carlier C. (1997). Les surveillants au parloir. Paris: Éditions de l'atelier.

20. La violence carcérale en question, rapport de recherche, GIP-justice, 2005.

21. Nous avons comparé les résultats obtenus, sur les axes interrogés, dans la zone des coursives avec d'autres espaces de travail (les parloirs, les lieux de soins, la porte, le mirador, le quartier mineur). 
Chauvenet, A., Orlic, F., \& Benguigui, G. (1994). Le monde des surveillants de prison. Paris: Presses universitaires de France.

Chauvenet, A., Orlic, F. Monceau, M., \& Rostaing, C. (2005). La violence carcérale en question, rapport de recherche. GIP-justice.

Claerhout, P. (2004). Émergence et régulation de la violence dans le paysage sonore pénitentiaire. In F. Courtine \& M. Renneville (eds.), La violence en prison, rapport de recherche (113-130). Agen: GIP justice, ENAP.

Diwo, R. (1997). Événements de vie, mentalisation, somatisation et tentatives de suicide: approche comparée à l'adolescence. Thèse de doctorat en psychologie, Université de Nancy 2, décembre 1997.

Enriquez, E. (2000). Le travail de mort dans les institutions. In R. Kaës (ed.), L'institution et les institutions (62-94). Paris: Dunod.

Foucault, M. (1975). Surveiller et punir. Paris: Gallimard.

Girard, R. (1982). Le bouc émissaire. Paris: Grasset.

Goffman, E. (1968). Asiles; études sur la condition sociale des malades mentaux. Paris: Les Éditions de Minuit.

Kaës, R. (2000). L'institution et les institutions. Paris: Dunod.

Kauffman, K. (1988). Prison officers and their world. Cambridge: Harvard University Press.

Lameyre, X. (2005). Actualité et acte de juger: éthique d'une poétique du procès. Petites Affiches, (138), 138-152.

Le Moigne, J-L. (1999). Modélisation des systèmes complexes. Paris: Dunod.

Levy, A. (2007). La peine: l'autre et le temps. Université de Haute Bretagne. Mémoire de Master 2.

Lhuillier, D., \& Aymard, N. (1997). L’Univers pénitentiaire. Du côté des surveillants de prison. Paris: Éditions Desclée de Brouwer.

Marty, P. (1991). Mentalisation et psychosomatique. Paris: Les empêcheurs de tourner en rond Éditeurs.

Mbanzoulou, P. (2000). La réinsertion sociale des détenus. De l’apport des surveillants de prison et des autres professionnels pénitentiaires. Paris: L'Harmattan.

Mercier, B. (2004). Dynamique et fonction de la mémoire des événements violents en milieu carcéral. In F. Courtine \& M. Renneville (eds.), La violence en prison, rapport de recherche (259-301). Agen: GIP justice, ENAP.

Ministère de la Justice (2008). Pratiques de Références opérationnelles, PRO, axe métier. Agen: Ministère de la Justice, ENAP.

Montandon, C., \& Crettaz, B. (1981). Paroles de gardiens, paroles de détenus; bruits et silences de l'enfermement. Genève: Masson - Médecine et Hygiène.

Morin, E., \& Le Moigne, J-L. (1999). L'intelligence de la complexité. Paris: L'Harmattan.

Moulin, V. (2008). Approche dynamique, processuelle et spatiale de l'agir criminel violent: vers une modélisation psychocriminologique intégrative du passage à l'acte. In B. Gaillard (ed.), La psychologie criminologique (83-98). Paris: In press. 
Rambourg, C. (2004).Violence médiatique: les personnels pénitentiaires dans la presse quotidienne nationale. In F. Courtine \& M. Renneville (eds.), La violence en prison, rapport de recherche (303-358). Agen: GIP justice, ENAP.

Rostaing, C. (1997). La relation carcérale. Paris: Presses Universitaires de France.

Silberman, M. (1995). A World of Violence. Corrections in America. Belmont (Californie): Wadsworth Publishing Company.

Siret, R. (2004). Les dimensions subjectives de la violence en prison du côté des surveillant(e)s. In F. Courtine \& M. Renneville (eds), La violence en prison, rapport de recherche (131-176). Agen: GIP justice, ENAP.

Vacheret, M. (2002). Relations sociales en milieu carcéral: une étude des pénitenciers canadiens. Déviance et société, 26 (1), 83-104.

Vacheret, M., \& Lemire, G. (2007). Anatomie contemporaine de la prison. Montréal: PUM.

Vasseur, V. (2000). Médecin-chef à la prison de la santé. Paris: Le cherche-midi.

Veil, C., \& Lhuillier, D. (2000). La prison en changement. Paris: Eres.

ABSTRACT - Based on observations revealing a trend among prison staff to invest positions away from the detention area, for the benefit of positions that do not involve working directly with inmates, this research seeks to understand this phenomenon. More precisely, the study from which this article is based consisted in analyzing what produces psychic conflict for detention officer when they are at work. From a modelling of psychological conflicts in the prison, this article details the identification of four professional positions and related psychic functions.

KEYWORDS - Prison establishment, prison officer, psychological conflicts, professional positioning.

RESUMEN - Luego de constatar que el personal de los centros de detención tiende a otorgar preferencia a los puestos que no incluyen contacto directo con los presos, esta investigación trata de explicar dicho fenómeno. En particular, el estudio en que se basa este artículo analiza el origen de los conflictos psíquicos del personal de los centros de detención relacionados con el ejercicio de sus funciones. A partir de la modelización de los conflictos psíquicos relacionados con la vigilancia en los pasillos de los establecimientos penitenciarios, el artículo define cuatro posicionamientos profesionales de los vigilantes y sus respectivos funcionamientos psicológicos.

PALABRAS CLAVE - Establecimiento penitenciario, vigilante, conflictos psíquicos, posicionamiento profesional. 